\title{
REPRESENTACIONES SOCIALES DEL CONTEXTO COMUNITARIO, ESCENARIO PARA GENERAR PRÁCTICAS DE ESCRITURA*
}

\author{
Beatriz Figueroa Sandoval ${ }^{1}$ \\ Mariana Aillon Neumann ${ }^{1}$ \\ Angie Neira Martínez ${ }^{1}$ \\ Lucía Ubilla Rosales ${ }^{2}$
}

\begin{abstract}
RESUMEN: Este trabajo plantea la necesidad de considerar las particularidades socioculturales para crear escenarios de aprendizaje donde las prácticas de lectura y escritura constituyan una herramienta de progreso académico. La muestra se conformó con 48 sujetos de secundaria de un colegio público chileno. Los datos fueron recogidos mediante escala tipo Likert y grupos focales. La información obtenida fue analizada cualitativamente a partir de la teoría de las Representaciones Sociales, permitiendo identificar elementos nucleares y periféricos de las creencias, opiniones, actitudes sobre su contexto. Los resultados arrojaron que los elementos periféricos de la representación social de los alumnos proporcionarían insumos para generar diseños didácticos que potencien los niveles de alfabetización.
\end{abstract}

Palabras claves: Lectura y escritura. Representación social. Contexto cultural.

\section{SOCIAL REPRESENTATION OF THE COMMUNITY SETTING, CONTEXT TO GENERATE WRITING PRACTICES}

\begin{abstract}
This paper raises the need to consider the socio-cultural features to create learning scenarios where reading and writing practices constitute a tool of academic achievement. The sample consisted of 48 high school students from a Chilean public school. Data were collected using a Likert-type scale and focus group. Obtained information was qualitatively analyzed considering the Social Representations theory; thus, core and peripheral elements related to beliefs, opinions and attitudes about the students' context were identified. Results showed that peripheral elements of students' social representation would provide inputs to generate didactic designs that would strengthen the literacy levels.
\end{abstract}

Keywords: Reading and writing. Social representation. Cultural context.

\footnotetext{
*Esta investigación forma parte del Proyecto Fondecyt Regular No 1140664: “Diseño multimodal en prácticas letradas escolares: avances desde los códigos restringidos a los elaborados" desarrollado desde marzo de 2014 a marzo de 2017 y es financiado por la Comisión Nacional de Investigación Científica y Tecnológica (CONICYT) de Chile.

${ }^{1}$ Universidad de Concepción, Facultad de Educación - Concepción, Chile. E-mails: bfiguero@udec.cl, maillon@udec.cl y angieneira@udec.cl

${ }^{2}$ Universidad Católica de Temuco, Facultad de Educación - Temuco, Araucanía, Chile. E-mail: lubilla@uct.cl DOI: 10.1590/ES0101-73302017160018
} 


\title{
REPRÉSENTATIONS SOCIALES DU MILIEU COMMUNAUTAIRE, SCÉNARIO POUR GÉNÉRER DES PRATIQUES D'ÉCRITURE
}

\begin{abstract}
RESSUME: Cet article soulève le besoin de penser aux particularités socioculturelles pour créer des scénarios $\mathrm{d}$ 'apprentissage où les pratiques de lecture et d'écriture constituent un véritable outil de progrès scolaire. L'échantillon a été constitué de 48 sujets d'enseignement secondaire qui appartiennent à un lycée publique Chilien. Les données ont été obtenues avec une échelle de type Likert et un groupe de discussion (focus group). L'information recueillie a été analysée qualitativement à partir de la Théorie des Représentations Sociales et a permis l'identification des éléments fondateurs et périphériques appartenant aux croyances, points de vue et attitudes envers leur milieu. Les résultats ont montré que les éléments périphériques de la représentation sociale des élèves fourniraient des apports pour créer du matériel didactique qui stimule les niveaux d'alphabétisation.
\end{abstract}

Mots-clés: Lecture et écriture. Représentation sociale. Contexte culturel.

\section{Introducción: visualización de la necesidad}

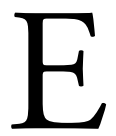

n las últimas décadas, en Chile, se han realizado numerosos esfuerzos por desarrollar las competencias de lectura y escritura en los diferentes niveles de escolaridad. De acuerdo con los informes de la Agencia Calidad de la Educación (2014; 2015), las pruebas estandarizadas nacionales e internacionales han demostrado que no existen avances significativos en dichas competencias. Además, estudios (MANZI et al., 2011) revelan las debilidades que presentan mayoritariamente los docentes respecto del dominio de los conocimientos disciplinarios del área del lenguaje, tanto en el manejo del currículum prescrito, como en la didáctica para enseñarlos. Este antecedente es relevante si consideramos que la didáctica articula y envuelve los conocimientos disciplinares, así como el manejo del currículo. Por lo tanto, un factor determinante está relacionado con el cómo los profesores gestionan la transposición didáctica (CHEVALLARD, 1991), atendiendo a las particularidades socioculturales de la escuela. Este complejo desafío implica el reconocimiento, apropiación y valoración del contexto cultural de los estudiantes para crear escenarios de aprendizaje en los cuales las prácticas de lectura y escritura incluyan el nivel pragmático, es decir, sean percibidas por los jóvenes como herramientas de progreso funcional en un tránsito desde su cultura hacia la cultura estándar y viceversa. 
Para lograr lo anterior, se requiere de propuestas didácticas que consideren articuladamente las competencias lecto/escritas con un anclaje socioeducativo. Tradicionalmente en Chile, estas tareas han sido abordadas por los profesores de manera intuitiva, sin mayores apoyos de constructos científicos más acabados, entre otras variables, por un apoyo técnico no siempre de buena calidad (BELLEI, 2010). Estos procesos requieren de instancias de reflexión sobre el porqué de las transformaciones pedagógicas, del análisis crítico de las propias experiencias de enseñanza.

Para avanzar en esta línea, se requieren estudios situados que permitan obtener claves desde una realidad geográfica y cultural específica respecto de cómo, desde la cultura emergente de los sujetos, considerada como punto de partida por medio de las prácticas de lectura y escritura, se logra una proyección hacia la cultura universal. En este sentido, entendemos como cultura emergente aquella que los niños construyen a partir de su contexto local — geográfico, social y familiar - y que es parte de su identidad como individuos y como grupo; mientras que concebimos la cultura universal como aquella constituida desde el contexto global, que incluye conocimientos sobre el mundo desde diversas dimensiones. La cultura universal se alcanza desde la escolaridad, a través del estudio y la comprensión de las distintas disciplinas del currículo.

\section{Nuestros Supuestos}

La consideración de la influencia de la cultura y de los contextos específicos en la producción escrita, por una parte, y de la explicación cognitiva de los procesos que intervienen, por otra, sitúan este estudio en un paradigma socio-cognitivo (DE BEAUGRANDE, 1997; VAN DIJK, 1994; WERTSCH, 1999; SALOMON, 1993). El aprendizaje y la alfabetización son actividades culturales e históricas, no solo porque se adquieren y cobran sentido a través de las interacciones sociales, sino también porque representan cómo un grupo cultural específico o una comunidad discursiva interpretan el mundo y luego genera información y conocimiento (BARTON et al., 2000). En este sentido, las diferencias socioculturales de los grupos a menudo están correlacionadas con las diferencias de dicha comunidad.

Las influencias socioculturales surgen debido a que los miembros de una comunidad determinada construyen su identidad como lectores a través de la forma de comportarse, de interactuar, de valorar, de pensar, hablar, leer y escribir (FREIRE Y MACEDO, 1987; GEE, 2005). La variación tiene directa relación con cómo se "lee el mundo", lo cual implica una ideología o concepción influida por la comunidad discursiva a la que se pertenece. A menudo, en sectores socialmente vulnerables, se suscitan conflictos porque los profesores, que son la voz 
oficial del currículo, imponen los discursos de un grupo dominante con el que no se sienten identificados los alumnos.

\section{Circunscribiendo el desafío}

Dado a lo anterior, es evidente la dificultad que los profesores de lenguaje enfrentan hoy para motivar las prácticas de lectura y escritura, situación que se acentúa cuando se trabaja con adolescentes. Las variables que se conjugan en el problema afectan siempre con mayores referencias a los sectores más desposeídos. La cuestión tiene que ver con cómo promovemos la escritura en jóvenes con un capital cultural disminuido en relación al estándar privilegiado por la escuela. Estos grupos, con experiencias de lenguaje centradas en la oralidad, se enfrentan al sistema escolar que busca instalar prácticas letradas desde una perspectiva formal externa, en muchos aspectos forzados, y siempre con déficit acumulativos. De esta forma, los escenarios de aprendizaje ${ }^{2}$ se configuran nadando contra la corriente, en circunstancias que sabemos que no existirán avances significativos, sino cuando la escritura surja con propiedad desde una motivación intrínseca del sujeto y en la medida en que este la movilice como herramienta funcional desde la pragmática de interacción social. ¿Cómo se construye con los estudiantes este puente desde su cultura de origen? y luego, ¿̇ómo se conjugan en un diseño didáctico las variables cognitivas y culturales que intervienen en el fenómeno de modo que se logren aprendizajes significativos?

\section{La propuesta en cuestión}

El objetivo del estudio propone diagnosticar el estado sociocultural que contextualiza las prácticas letradas de un grupo de jóvenes de séptimo año de una escuela de la comuna de Hualpén, articulando las necesidades requeridas por el currículo escolar con la realidad de la cultura emergente. Se pretende alcanzar un conocimiento de la cultura de los jóvenes mediante un diseño multimodal de escritura $^{3}$. El grupo total de la muestra está compuesto por tres cursos de séptimo año básico con un total de 120 estudiantes. Se trata de una escuela municipal con una población escolar que presenta un índice de vulnerabilidad social (IVE) de un $72 \%$, lo que implica que los estudiantes pertenecen a familias de bajos ingresos, cuyos padres alcanzaron el nivel secundario de escolaridad.

\section{Metodología de Investigación}

Desde la dimensión cualitativa, se busca identificar los elementos básicos que componen la identidad cultural del grupo de estudiantes intervenido. 
El modelo investigativo seleccionado es la teoría de las representaciones sociales (MOSCOVICI, 1961) por cuanto nos proporciona la posibilidad de dar cuenta de los aspectos socio-cognitivos que indagamos. Del grupo en pleno, 120 sujetos, se consideró una sub-muestra conformada aleatoriamente por 48 niños de los tres cursos de séptimo año de la escuela. Por tratarse de una investigación cualitativa, no es necesario trabajar con la muestra completa, puesto que el estudio en profundidad de las representaciones contemplaba la realización de grupos focales, requiriendo una muestra menor, compuesta por alumnos de los diferentes cursos.

Los datos fueron recogidos a partir de dos instrumentos: un cuestionario compuesto por una escala Likert, aplicado on line con la aplicación Formulario de Google Drive, y tres grupos focales con una entrevista semi-estructurada.

\section{Aproximación a la teoría de las representaciones sociales (rs)}

La teoría de las representaciones sociales considera el cambio paradigmático propuesto por el constructivismo respecto de la inexistencia de la realidad objetiva, planteando un análisis sobre cómo los individuos de una comunidad particular, a partir de la relación sujeto/objeto, parte intrínseca del vínculo social, se apropian y reestructuran la realidad mediante la integración de las características del objeto desde sus experiencias; así como del sistema de normas y actitudes generados en torno de este (ABRIC, 2001).

El concepto de rs designa una forma de conocimiento específico, un saber de sentido común, cuyos contenidos manifiestan la operación de procesos generativos y funcionales socialmente caracterizados. Por lo tanto, constituyen modalidades de pensamiento práctico orientados hacia la comunicación, la comprensión y el dominio del entorno social, material e ideal (JODELET, 1999).

Visto lo anterior, interesa conocer las representaciones que los sujetos de la muestra poseen respecto de su comuna para identificar desde estas los núcleos culturales que los identifican y definen, para luego asentar desde estos las propuestas de escritura.

\section{Las fases del proceso investigativo}

El proceso de definición de las rs de los estudiantes se llevó a cabo a partir de tres etapas:

1. Identificación del contenido de la representación,

2. Categorización jerárquica de los elementos que la componen,

3. Determinación del núcleo o centro de la representación, así como de sus elementos periféricos. 
1. Identificación del contenido de la representación. En esta fase privilegiamos la técnica de recolección denominada grupo focal. Las conversaciones se organizaron en tres grupos de dieciséis estudiantes cada uno y se dialogó respecto de temas relacionados con su comuna.

Del análisis de los datos se determinó que, dentro del contenido de la representación de la comuna, se identifican los siguientes hitos temáticos recurrentes en el discurso de los alumnos:

- Presencia significativa de una configuración espacial del entorno inmediato compuesta por elementos concretos: casa- pasaje, barrio, plazas, jardines, canchas, sedes sociales, colegio, autoridades como PDI (Policía de Investigaciones) y Carabineros;

- Presencia de una configuración espacial más difusa de la periferia compuesta por elementos menos concretos: industrias de la comuna de Hualpén y salidas al mar de la comuna (Desembocadura del Bío Bío y Caleta Lenga);

- Presencia de prácticas específicas y recurrentes en torno al uso de las tecnologías de la información (internet) y de los medios masivos de comunicación (las redes sociales y el cine);

Con el fin de triangular la información, se aplicó una escala tipo Likert para verificar si estos núcleos temáticos efectivamente adquieren, en el discurso de los estudiantes, la misma relevancia que la obtenida en los grupos focales.

2. Categorización jerárquica de los elementos constitutivos de la representación: En esta etapa, se categorizaron los focos temáticos ya identificados, integrando la información obtenida de los grupos focales con la escala Likert. Este proceso se orientó hacia la búsqueda del núcleo central de la representación, estableciendo las categorías o subconjuntos temáticos o actitudinales que constituyen la estructura de la misma.

El resultado de este análisis define un esquema que contiene tres tópicos centrales en torno a los cuales se definen las rs: Entorno inmediato cotidiano, Entorno periférico y Espacios virtuales. Nótese que los dos primeros corresponden a espacios territoriales y el tercero definido como un espacio virtual, se articula en torno al uso de los medios de comunicación como teléfono e internet.

El entorno inmediato cotidiano constituye el núcleo de la rs y está conformado por su percepción positiva del barrio, donde los jóvenes viven junto a sus familias y han establecido lazos afectivos de convivencia y amistad. Los elementos que forman parte de este entorno son:

- Las plazas, áreas verdes y jardines que integran la idea de los estudiantes respecto de que habitan una comuna de espacios agradables, limpia y 
ordenada, como si se tratase de un barrio de clase media. Un espacio seguro que promueve la convivencia al aire libre;

- Las multicanchas y sedes sociales que conforman puntos de reunión con los amigos y vecinos para realizar actividades deportivas, celebrar efemérides e incluso recibir capacitación, tales como cursos de cocina, tejido, música, entre otros;

- La municipalidad que proporciona información y recursos relacionados con el bienestar material de las familias de la comuna. Esta institución organiza y promueve actividades de convivencia, por ejemplo: celebraciones de navidad, fiestas patrias, pascua de los conejos, concierto del día de los enamorados, etc.;

- El consultorio que provee atención de salud básica para las familias de los estudiantes: casos de urgencia, atención dental y pediátrica;

- La escuela que goza de prestigio por su nivel destacado en el contexto comunal y ha proporcionado figuras reconocidas a nivel nacional en el ámbito deportivo, específicamente futbolístico.

El entorno periférico esporádico se refiere a la representación que los sujetos tienen de los lugares, actividades e instituciones que juegan un rol secundario y a veces ocasional en sus vidas. Por lo mismo, son elementos que se encuentran más alejados del núcleo de la representación constituida por el barrio, entre estas destacan:

- Las industrias petroquímicas y de acero que se encuentran en la comuna y son una fuente laboral importante para los profesionales, ingenieros y técnicos calificados dentro de la región. Sin embargo, los estudiantes muestran una percepción negativa de estas fuentes laborales, a las cuales califican de riesgosas y mal remuneradas;

- Los museos, caletas de pescadores y desembocadura del Bío-Bío son lugares de recreación visitados ocasionalmente por los estudiantes y sus familias. El conocimiento que tienen de estos ambientes naturales y culturales está ligado a saberes populares de historia, geografía y ecología;

- Internet (wi fi) y los dispositivos (computadores y celulares) con aplicaciones de redes sociales a las cuales tienen acceso en sus casas y, principalmente, en su escuela. En el Cuadro 1, se sintetiza la información antes descrita.

\section{Determinación y el control del núcleo central}

La tercera fase permitió la determinación del objeto mismo de la representación a partir de la identificación de su núcleo, verificando los resultados obtenidos 


\section{Cuadro 1}

Representaciones sociales sobre Hualpén recogidas en grupos focales y cuestionarios.

\begin{tabular}{|c|c|c|c|}
\hline Tópicos & $\begin{array}{l}\text { Núcleos/ } \\
\text { Elementos }\end{array}$ & $\begin{array}{l}\text { Representaciones } \\
\text { grupos focales }\end{array}$ & $\begin{array}{l}\text { Representaciones } \\
\text { cuestionarios }\end{array}$ \\
\hline \multirow{8}{*}{$\begin{array}{l}\text { Entorno } \\
\text { inmediato } \\
\text { cotidiano }\end{array}$} & \multirow[b]{2}{*}{$\begin{array}{l}\text { Casa-pasaje, } \\
\text { representan espacios } \\
\text { abiertos y cerrados) }\end{array}$} & $\begin{array}{l}\text {-Tranquilidad. No existe } \\
\text { delincuencia ni tráfico } \\
\text { vehicular. }\end{array}$ & $\begin{array}{l}\text {-Habitabilidad. } \\
\text { Recomendarían su barrio para } \\
\text { vivir ( } 81 \% \text { Muy de acuerdo) }\end{array}$ \\
\hline & & $\begin{array}{l}\text {-Peligrosidad en } \\
\text { algunos sectores por: } \\
\text { tráfico de drogas, } \\
\text { robos, ajusticiamientos, } \\
\text { polución (industrias). }\end{array}$ & $\begin{array}{l}\text {-Preocupación por existencia } \\
\text { de tráfico de drogas y robo } \\
\text { ( } 74 \% \text { Muy de acuerdo) }\end{array}$ \\
\hline & $\begin{array}{l}\text { Plazas y/o áreas } \\
\text { verdes, jardines }\end{array}$ & $\begin{array}{l}\text {-Recreación: juegos, } \\
\text { andar en bicicleta y } \\
\text { patineta. } \\
\text {-Epicentro de la } \\
\text { interacción social: } \\
\text { celebración eventos y } \\
\text { efemérides. }\end{array}$ & $\begin{array}{l}\text {-Esparcimiento. Lugar de } \\
\text { paseo y de juegos ( } 92 \% \text { Muy } \\
\text { de acuerdo) }\end{array}$ \\
\hline & Multicanchas & $\begin{array}{l}\text { Celebración de } \\
\text { efemérides: día de la } \\
\text { independencia, juegos. }\end{array}$ & $\begin{array}{l}\text {-Práctica de deporte y vida } \\
\text { al aire libre ( } 94 \% \text { Muy de } \\
\text { acuerdo) }\end{array}$ \\
\hline & Municipalidad & $\begin{array}{l}\text { Organización } \\
\text { de actividades y } \\
\text { celebraciones: navidad, } \\
\text { pascua de los conejos. }\end{array}$ & $\begin{array}{l}\text {-Entrega de servicios } \\
\text { y beneficios de salud, } \\
\text { alimentación, becas, bonos, } \\
\text { vivienda a las familias: ( } 81 \% \\
\text { Muy de acuerdo) } \\
\text {-Necesidad de informarse. } \\
\text { (100\% muy de acuerdo) }\end{array}$ \\
\hline & Sedes sociales & $\begin{array}{l}\text { Oferta de cursos abiertos } \\
\text { a la comunidad: cocina, } \\
\text { música, etc. }\end{array}$ & $\begin{array}{l}\text {-Desarrollo de actividades } \\
\text { recreativas para toda la familia } \\
\text { ( } 78 \% \text { muy de acuerdo) } \\
\text {-Participación pasiva de } \\
\text { vecinos (solo asistencia) } \\
\text { en actividades. ( } 50 \% \text { ni de } \\
\text { acuerdo ni en desacuerdo) }\end{array}$ \\
\hline & Colegios & $\begin{array}{l}\text { Influencia en modelo } \\
\text { profesional: ex-alumnos } \\
\text { futbolistas famosos en el } \\
\text { extranjero. }\end{array}$ & $\begin{array}{l}\text {-Valoración de la calidad de } \\
\text { la educación impartida. ( } 97 \% \\
\text { muy de acuerdo) } \\
\text {-Proyección importante en la } \\
\text { identidad de los alumnos. }\end{array}$ \\
\hline & $\begin{array}{l}\text { Hospitales y } \\
\text { consultorios }\end{array}$ & $\begin{array}{l}\text { Influencia en modelo } \\
\text { profesional: médico. }\end{array}$ & $\begin{array}{l}\text {-Satisfacción de necesidades } \\
\text { básicas de salud de los vecinos } \\
\text { ( } 91 \% \text { muy de acuerdo) }\end{array}$ \\
\hline
\end{tabular}




\section{Cuadro 1}

Continuação.

\begin{tabular}{|c|c|c|c|}
\hline Tópicos & $\begin{array}{l}\text { Núcleos/ } \\
\text { Elementos }\end{array}$ & $\begin{array}{l}\text { Representaciones } \\
\text { grupos focales }\end{array}$ & $\begin{array}{l}\text { Representaciones } \\
\text { cuestionarios }\end{array}$ \\
\hline & $\begin{array}{l}\text { Industrias: } \\
\text { Enap, Petrox, } \\
\text { Inchalam, Cementos } \\
\text { Biobío, Coca Cola }\end{array}$ & $\begin{array}{l}\text {-Rechazo. Fuente } \\
\text { laboral mal remunerada } \\
\text { y riesgosa (peligro } \\
\text { accidentes). }\end{array}$ & $\begin{array}{l}\text {-Desinterés por la industria } \\
\text { como fuente de ingreso } \\
\text { familiar. ( } 60 \% \text { en desacuerdo } \\
\text { o no sabe; } 40 \% \text { de acuerdo). }\end{array}$ \\
\hline $\begin{array}{l}\text { Entorno } \\
\text { periférico }\end{array}$ & $\begin{array}{l}\text { Ambiente natural } \\
\text { caleta Lenga, } \\
\text { desembocadura } \\
\text { Biobío, Museo Pedro } \\
\text { del Río Zañartu }\end{array}$ & $\begin{array}{l}\text { Distracción de fin de } \\
\text { semana: comida o } \\
\text { almuerzo en restaurantes, } \\
\text { juegos, caminatas en } \\
\text { cerros y playas no aptas } \\
\text { para el baño, pesca, } \\
\text { visita al museo, rodeos } \\
\text { y ramadas, observación } \\
\text { astronómica. }\end{array}$ & $\begin{array}{l}\text {-Interés por conocer museos } \\
\text { ( } 92 \% \text { Muy de acuerdo) } \\
\text { visitar y preservar humedales } \\
\text { ( } 82 \% \text { Muy de acuerdo) } \\
\text { disfrutar del turismo en caleta } \\
\text { Lenga ( } 95 \% \text { Muy de acuerdo) } \\
\text { y pasear por desembocadura } \\
\text { ( } 89 \% \text { Muy de acuerdo). }\end{array}$ \\
\hline $\begin{array}{l}\text { Espacios } \\
\text { virtuales } \\
\text { TIC, } \\
\text { medios } \\
\text { masivos } \\
\text { de c. }\end{array}$ & $\begin{array}{l}\text { Internet en el } \\
\text { colegio (sala } \\
\text { computación y wi fi), } \\
\text { la casa y dispositivos } \\
\text { personales (celulares) }\end{array}$ & $\begin{array}{l}\text { Apoyo para realizar tareas. } \\
\text {-Interacción a través de } \\
\text { aplicaciones sociales: } \\
\text { Facebook, Whatsapp, } \\
\text { Retrica. }\end{array}$ & $\begin{array}{l}\text {-Valoración de computador } \\
\text { en el hogar ( } 97 \% \text { Muy de } \\
\text { acuerdo) } \\
\text {-Utilización de Internet como } \\
\text { principal herramienta de } \\
\text { trabajo escolar ( } 97 \% \text { Muy de } \\
\text { acuerdo) } \\
\text {-Reconocimiento del uso } \\
\text { de medios audiovisuales } \\
\text { como recurso favorito de } \\
\text { sus profesores ( } 90 \% \text { Muy de } \\
\text { acuerdo) } \\
\text {-Privilegio de redes sociales } \\
\text { como medio de interacción } \\
\text { (81\% Muy de acuerdo). } \\
\text {-Preferencia por aplicaciones } \\
\text { de pasatiempo en los } \\
\text { smartphones ( } 78 \% \text { Muy de } \\
\text { acuerdo). }\end{array}$ \\
\hline
\end{tabular}

en las etapas precedentes, en particular, las hipótesis de centralidad de tal o cual elemento. El proceso se ejecutó con la técnica del cuestionario para identificar el núcleo central, elemento que genera y estructura las concepciones de los estudiantes respecto de los tres tópicos abordados en los puntos anteriores es la idea de barrio. Su determinación, como núcleo de la representación, está avalada principalmente por las funciones que desempeńa respecto del resto de los elementos, puesto que cumple con:

- Una función generadora: El barrio es el elemento mediante el cual se crea y se transforma la significación de los otros elementos consti- 
tutivos de la representación. Cuando los estudiantes son consultados respecto de las características del lugar de residencia próximo y periférico en el que viven, las descripciones inmediatas apuntan al barrio, su definición territorial, el tipo de viviendas, los espacios comunes como plazas, jardines. Y se explayaron sobre todo en la descripción de la vida al interior del barrio como un territorio natural y cultural que los identifica y define;

- Una función organizadora: Desde el barrio como espacio territorial central, comienzan a surgir los elementos periféricos estableciendo con aquel la naturaleza de los lazos que los unen. Mencionan, por ejemplo, las diversas actividades que se desarrollan en la sede municipal, los paseos familiares a lugares naturales recreativos, la escuela y las actividades en que participa la familia. De tal forma, el barrio constituye el elemento unificador y estabilizador de la representación;

- Una función de pertenencia: colectivamente los sujetos reconocen el barrio como de su propiedad y lo re-presentan como un espacio con características, en algunos casos idealizadas, es decir, privilegiando la descripción expresiva focalizada en destacar los aspectos positivos de su entorno, relevándolos sobre los elementos negativos. Por ejemplo, se mencionaron hechos de delincuencia de manera tangencial, sin relevarlo, a pesar de que objetivamente se trata de un barrio con altos índices de delincuencia. En cambio, espacios de encuentro, como la plaza y centros comunitarios, son altamente valorados por los estudiantes. Por lo tanto, el barrio constituye el elemento más estable de la representación, el que garantiza su perennidad respecto de contextos más móviles y evolutivos, como el caso de los espacios recreativos o industriales (ABRIC, 2001).

\section{Esquema de la organización y estructura de las representaciones sociales}

El esquema de la organización interna de las rs de los estudiantes, descrito anteriormente, sigue el modelo de la hipótesis del núcleo central de Abric (2001), quien plantea que toda rs está organizada en torno a un núcleo compuesto por uno o varios elementos, como se observa en la Figura 1.

Como se aprecia en el esquema, la centralidad del núcleo determina la aparición de ciertos elementos periféricos que se organizan, y adquieren su valor y función en relación con él. Estos elementos constituyen el aspecto más concreto de la representación, en tanto: "Abarcan informaciones retenidas, seleccionadas e interpretadas, juicios formulados al respecto del objeto y su entorno, estereotipos y creencias" (ABRIC, 2001, p. 23). Estos elementos periféricos 
presentan una jerarquización, aquellos que están más próximos al núcleo, cumplen un papel relevante en la concreción del significado de la representación. En cambio, los elementos más distantes desempeñan la función de ilustrar, aclarar, justificar dicha significación.

\section{Análisis de las representaciones sociales de los jóvenes respecto de su comuna}

Considerando que las representaciones sociales son un conjunto organizado y jerarquizado de informaciones, creencias, opiniones y actitudes respecto de un objeto (ABRIC, 2001), el análisis de este conjunto requirió de la identificación de su contenido y estructura, desde una doble lógica: cognitiva y social.

En efecto, cada sujeto de la muestra presenta un perfil psicológico que rige sus procesos cognitivos, los cuales están condicionados por aspectos tales como: la etapa adolescente en que se encuentran (12 y 13 años de edad), los niveles de desarrollo de las competencias curriculares como: habilidades de lectura, escritura, comunicación oral, de resolución de problemas, estilos de aprendizaje, entre otros. A su vez, la cognición está determinada por condiciones sociales, en este caso, los estudiantes en su mayoría provienen de familias vulnerables, con padres que trabajan fuera de la región, y viven en los barrios aledaños a la escuela, algunos de los cuales registran altos niveles de violencia y delincuencia.

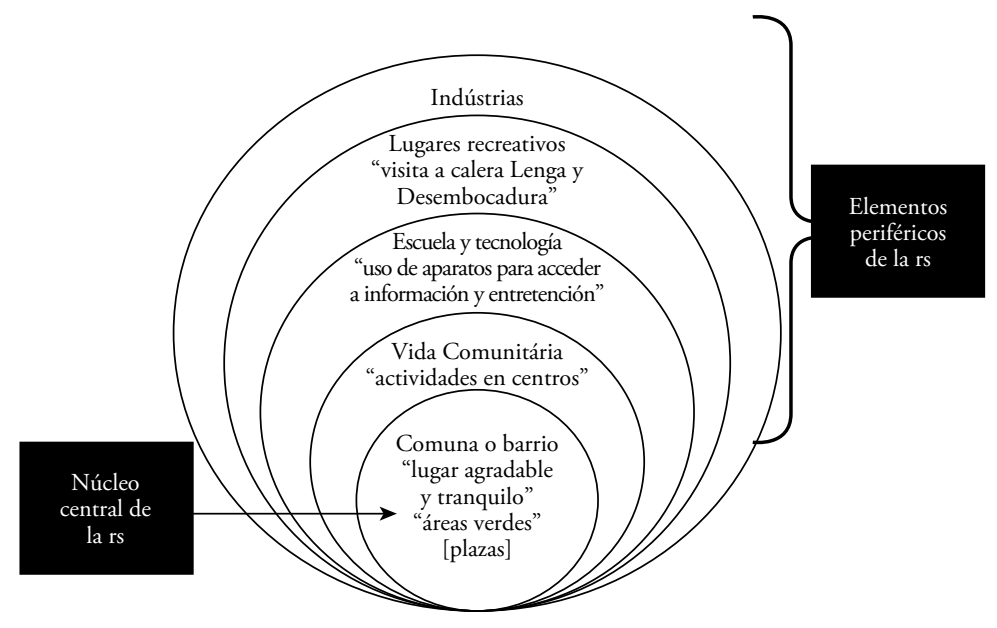

Figura 1

Esquema de la organización interna de las rs de los estudiantes. 
Para nuestro estudio final, que pretende elaborar un diseño didáctico de escritura en función de la premisa que cognición y lenguaje son un constructo intelectual indivisible, la dimensión cognitiva está acotada a los desempeños de las competencias comunicativas orales y escritas. Por tanto, en este análisis interesa la perspectiva que condiciona la producción discursiva, considerada como elemento visible de la dimensión cognitiva de los sujetos.

1. El contexto discursivo a partir del cual se obtuvo la información se delimita por:

- Condiciones de producción: participación de 48 estudiantes en un grupo focal con investigadores de la Facultad de Educación de la Universidad de Concepción que entablan una entrevista semi-estructurada acerca de temas relacionados con el lugar donde viven, los sujetos, sus experiencias y la valoración del mismo.

- Auditorio: investigadores de la UdeC, quienes realizan preguntas y registran las respuestas y comentarios de los estudiantes de $7^{\circ}$ año de los cursos A, B y C.

- Situación: pública formal en la medida que los estudiantes dialogan con "visitas" que han llegado al establecimiento autorizados por los directivos. Aun cuando existe espontaneidad en el transcurso de esta entrevista colectiva, los sujetos se comportan de acuerdo con un protocolo básico, propio de interacciones con adultos "extrańos" o "forasteros", profesionales, a quienes deben causar una buena impresión.

- Propósito: los sujetos de la muestra enfrentados a la situación descrita anteriormente, demuestran interés por argumentar y convencer a las "visitas" acerca de las bondades de su "barrio", presentándolo como un lugar agradable para vivir, lo cual se evidencia en sus comentarios.

2. Contexto social. Es el más general de la representación, donde intervienen las relaciones simbólicas propias de un campo social referidas a:

El contexto ideológico que se expresa en la valoración de los estudiantes acerca de su barrio. Desde la perspectiva social, aprecian el contar con áreas verdes y jardines en los sectores donde viven y estudian, vinculando estos elementos con la idea de "orden" y "limpieza", que caracteriza a la clase media. Asimismo, esta dimensión se asocia con la "seguridad" de los barrios y la ocurrencia "esporádica" o "eventual" de actos delictivos. Los jóvenes destacan la importancia de la convivencia de los vecinos en las variadas actividades ofrecidas en los centros comunitarios y por la municipalidad. 
Desde la perspectiva económica, se releva el acceso a la tecnología, como el uso de teléfonos celulares y computadores conectados a Internet; y la oportunidad de ir al cine cuando los padres que trabajan en la zona norte del país regresan con días de descanso.

Los sujetos valoran la oportunidad de estudios proporcionada por la escuela con la que se identifican positivamente y desde la cual se proyectan hacia un futuro profesional y laboral, donde explicitan la inconveniencia de trabajar en las industrias que pertenecen a la comuna por los bajos sueldos que ofrecen. Un número importante de varones manifiesta interés por seguir una carrera deportiva profesional que tiene de modelo a reconocidos jugadores de fútbol que crecieron en el lugar.

Desde la perspectiva cultural, destaca el juicio negativo hacia programas de la televisión dedicados a las noticias y farándula. Las razones son referidas a la baja credibilidad y manipulación de la información, como al tiempo invertido en temas intrascendentes y escandalosos, respectivamente.

El lugar ocupado en el sistema social privilegia la imagen del hijo de una familia: i. esforzada con un jefe de hogar que viaja fuera de la región para trabajar; ii. ordenada porque tiene reglas y control sobre las actividades de los más jóvenes, estableciendo horarios y acceso a sitios de internet y programas de televisión; iii. interesada en la educación y en los modelos a seguir, optando por una escuela distante de su casa para recibir educación de calidad.

\section{Discusión de los resultados encontrados}

En síntesis, la $r$ del barrio identificada en los estudiantes de la muestra cumple con la función de concreción desde la perspectiva social, en la medida que ancla a los jóvenes a la realidad y les permite revestirla en términos concretos, comprensibles y transmisibles. Los elementos secundarios o periféricos surgen para especificar este núcleo, describir sus detalles y crear un ambiente contextual que lo expande. El barrio es el lugar donde se sitúa la representación que denominamos el entorno inmediato cotidiano, especificado en el discurso de los sujetos cuando mencionan, por ejemplo, la presencia de: plazas, sedes sociales, multicanchas, etc.

A su vez, los elementos periféricos, como los lugares de recreación y las industrias, con descripciones más flexibles que las de los elementos centrales, cumplen con la función de regulación, es decir, desempeñan el papel de adaptar la representación a las evoluciones del contexto. Así, por ejemplo, las opiniones respecto de los aportes de la zona industrial a la comuna fueron negativas aunque con el reconocimiento de los aportes de estas al contexto nacional, pero no así al cotidiano inmediato, constituyéndose en un aspecto móvil y evolutivo de la representación. 
La función reguladora, además, asume un criterio temporal y responde a la pregunta ¿cuándo? Esta dimensión fue identificada en el momento en que los sujetos establecieron la diferencia entre un antes/ahora respecto, por ejemplo, de tópicos como la violencia ("antes había más violencia y drogas en el barrio"), las áreas verdes ("las plazas antes tenían menos árboles, ahora han crecido y los jardines se cuidan más"), el uso de la tecnología ("antes usábamos Instagram, ahora usamos Retrica"), entre otros aspectos. De esta forma, el núcleo central de la representación resiste al cambio, puesto que su transformación ocasionaría un trastorno completo en la medida que desdibuja los aspectos positivos e ideales con que caracterizan su barrio. Por tanto, el sistema periférico funciona como el sistema de defensa de la representación y constituye lo que Flament (1987) llama su "parachoques». Por esta razón, la transformación de una representación opera en la mayoría de los casos mediante la transformación de sus elementos periféricos observada en cambio de ponderación, las interpretaciones nuevas, las deformaciones funcionales defensivas, la integración condicional de elementos contradictorios. En este sentido, en el sistema periférico, las contradicciones podrán aparecer entonces y ser sostenidas.

Una muestra del efecto de "parachoques» quedó de manifiesto cuando los estudiantes señalaron mayoritariamente, por ejemplo, que su barrio es seguro. Sin embargo, en el transcurso de la entrevista algunos de ellos mencionaron que ha habido algunos asaltos con características violentas cerca de sus hogares y algunos problemas de drogas. En ciertos comentarios, los actos violentos se identificaron mediante la atribución de su presencia en otros barrios un poco más lejanos y emblemáticos respecto de los territorios más próximos de los informantes.

\section{Avances de la propuesta didáctica generada desde las representaciones}

El objetivo, ahora, es utilizar esta construcción para definir un diseño multimodal de escritura que, por una parte, considere el contexto social, es decir, el contexto ideológico de la representación y, por otra, permita al sujeto desde su discurso como expresión cognitiva, operacionalizada en un género específico, mostrar el lugar que como individuo ocupa en este sistema social. Como hemos señalado una representación única, organizada alrededor de un núcleo central, puede dar lugar a diferencias aparentes, relacionadas con la apropiación individual o con contextos específicos que son traducidos por sistemas periféricos y eventualmente por comportamientos relativamente diferentes. Por supuesto, a condición que esas diferencias sean compatibles con un mismo núcleo central (ABRIC, 2001).

En consecuencia, utilizaremos para anclar la producción escrita los tópicos pertenecientes a elementos periféricos como las industrias de la comuna: 
Enap, Petrox, Inchalam, Cementos Biobío, Coca Cola, así también los espacios recreativos como: Lenga, desembocadura del río Biobío, Museo Pedro del Río Zańartu. Además, destacaremos como elemento transversal las Tics para potenciar la producción desde una plataforma virtual, aprovechando que los estudiantes manifiestan interés en el trabajo con tecnología e Internet.

Los géneros seleccionados para las producciones son: discursos del comentario y reportajes, que desarrollan formas argumentativas y expositivas presentes en los contenidos curriculares de séptimo año.

Entonces, el diseño multimodal de escritura buscará que los estudiantes tomen conciencia de que son generadores de signos y deberá satisfacer tres demandas básicas:

1. representar las situaciones o acontecimientos que ocurren en su realidad local para, desde esta, transitar a la realidad global, lo que sería la función ideacional;

2. representar las relaciones sociales entre los participantes en el proceso de comunicación escrita que implica la definición de la situación de enunciación (tema, destinatario y propósito), lo que sería la función interpersonal, $\mathrm{y}$

3. representar todo eso como una entidad-mensaje, es decir, como un «texto» internamente coherente en consonancia con su cultura, lo que sería la función textual (KRESS, 2005).

\section{Conclusiones y proyecciones}

Los hallazgos del estudio precedente nos permiten, a partir de la representación recabada, programar la escritura para los estudiantes de manera que estos lleguen a producir textos en los que:

- Manifiesten una opinión (respuesta) expresada respecto de un objeto o situación y que dicha opinión sea parte constitutiva del objeto o situación aludida, por lo tanto, determine su existencia. Esta visión global y unitaria del objeto, lo será al mismo tiempo del sujeto, como parte de una representación consistente;

- Expresen un carácter social al formar parte intrínseca de la relación entre un objeto y un sujeto que comparten con su grupo de pertenencia un sistema de valores, situado histórica y culturalmente. En este sentido, el escrito será un producto de una concepción ideológica, con un fuerte sentido identitario; 
- Constituyan un marco de referencia que guíe tanto las prácticas sociales del propio individuo como las de su grupo, permitiéndoles definir e incluso adaptar su lugar en el mundo. Esperamos que las producciones actúen luego como un "sistema de pre-decodificación" de la realidad determinando un conjunto de anticipaciones y expectativas respecto de esta (ABRIC, 2001);

- Reflejen un autor situado en el mundo, en la medida en que defina una "toma" de posición que le permita interpretar las conductas y la realidad, de acuerdo con el marco de referencia que comparte con su grupo de pertenencia;

- Los textos aporten a la reestructuración de la realidad con una visión funcional que posibilite la integración de las características del objeto, de las experiencias anteriores del sujeto y de su sistema de normas y actitudes (ABRIC, 2001).

Al cumplir con las premisas anteriores desde la didáctica de la lengua, esperamos construirles a los jóvenes de Hualpén una propuesta pertinente que contextualice sus prácticas de escritura, estableciendo un nexo entre su cultura de origen local y su progreso hacia la cultura universal. Estos antecedentes constituyen un marco que permite comprender de qué modo los aprendices construyen su competencia escrita, conjugando las variables cognitivas y culturales que intervienen en el proceso, relacionando los contenidos de aprendizaje con las actividades de producción en contextos que les otorgan sentido (CAMPS, 2001). Insistimos que la creación de los diseños didácticos multimodales cumple con las transformaciones para desarrollar la competencia de escritura, de acuerdo a los estándares del currículo nacional.

\section{Notas}

1. El término capital cultural empleado en este trabajo está tomado de Bourdieu (1997) y se refiere al conjunto de elementos simbólicos tales como: habilidades, gustos, pertenencias materiales que una persona adquiere debido al hecho de pertenecer a una clase social en particular. El compartir formas similares de capital cultural crea un sentido de identidad colectiva y posición de grupo, lo que se conoce como "personas como nosotros". Este sentido de identidad colectiva es brindado en primer lugar por la familia que transmite su capital cultural. Muchas veces la cultura familiar y la cultura de la escuela son antagónicas.

2. Concebimos un escenario de aprendizaje como un conjunto de actividades, recursos y métodos que refleja una unidad de aprendizaje. Un escenario puede representar, además, una situación de aprendizaje manifestada en una lección a través de la definición de roles, actividades, recursos y herramientas (KOPER; OLIVIER, 2004). 
3. En el modelo de alfabetización multimodal (KRESS, 2005; JEWITT, 2008), se define diseño como un proceso dinámico de implementación de los recursos que las personas utilizan de acuerdo a necesidades de comunicación específicas, los cuales están referidos al manejo de diversos modos o códigos semióticos (código verbal, la imagen, los colores, el sonido, entre otros). Este modelo estimula a los alumnos a explorar la producción de innovación y cambio. Estos diseños pueden estar referidos tanto a prácticas de lectura como de escritura.

\section{Referencias}

ABRIC, J. (Org.). Prácticas Sociales y Representaciones. México: Ediciones Coyoacán, 2001. AGENCIA CALIDAD DE LA EDUCACIÓN. Informe Nacional Resultados Chile Pisa 2012. Santiago: Ministerio de Educación y OECD, 2014. Disponible en: < http://archivos. agenciaeducacion.cl/Informe Nacional Resultados Chile PISA 2012.pdf>. Accesso en: 12 abr. 2017.

Resultados Simce Comprensión de Lectura y Matemática 6. ${ }^{\circ}$ básico 2013. Santiago: Agencia Calidad de La Educación, 2015. Disponible en: < http://archivos. agenciaeducacion.cl/documentos-web/Informe Nacional Resultados Simce2013.pdf >. Acceso en: 12 abr. 2017.

BARTON, D.; HAMILTON, M.; IVANIC, R. Situated Literacies. London and New York: Routhledge, 2000.

BELLEI, C. (Coord.). Asistencia Técnica Educativa: ¿aporte al mejoramiento escolar? Santiago: Ocholibros, 2010.

BOURDIEU, P. The Forms of Capital. In: HALSEY, A.H. et al. (Eds.). Education: Culture, Economy and Society. Oxford, England: Oxford University Press, 1997. p. 46-58.

CAMPS, A. El aula como espacio de investigación y reflexión: investigaciones en Didáctica de la Lengua. Barcelona: Graó, 2001.

CHEVAlLARD, I. La transposition didactique. París: La Pensee Saivage, 1991.

DE BEAUGRANDE, R. New Foundations for a Science of Text and Discourse. Greenwich, CT: Ablex, 1997.

FLAMENT, C. Pratiques et representations sociales. In: BEAUVOIS, J.; JOULE, V.; MONTEIL, J. (Orgs.). Perspectives cognitives et conduites sociales. Cousset: Del Val, 1987.

FREIRE, P.; MACEDO, D. Reading the word and the world. South Hadley Mass.: Bergin and Garvey, 1987.

GEE, J.P. La ideología en los discursos. Madrid: Morata, 2005.

JEWITT, C. Multimodal Discourses across the Curriculum. In: MARTINJONES, M.; MEJIA, A.M.; HORNBERGER, N.H. (Orgs.). Encyclopedia of Language and Education: Discourse and Education. 2a ed. Philadelphia: Springer, 2008. v. 3. p. 357-367.

JODELET, D. (Org.). Les Représentations sociales. Paris: Presses Universitaires de France, 1999. 
KOPER, R.; OLIVIER, B. Representing the Learning Design of Units of Learning. Educational Technology \& Society, v. 7, n. 3, p. 97-111, 2004.

KRESS, G. El alfabetismo en la era de los nuevos medios. Málaga: Aljibe, 2005.

MANZI, J.; GONZÁLEZ, R.; SUN, J. (Orgs.). La evaluación docente en Chile. Santiago: MIDENUC, Centro de Medición, 2011.

MOSCOVICI, S. La psychanalyse, son image et son public. Paris: PUF, 1961.

SALOMON, G. Distributed cognitions. New York: Cambridge University Press, 1993.

VAN DIJK, T. Discurso, Poder y Cognición Social. Conferencias Maestría en Lingüística. Cuadernos, Colombia, n. 2, v. 2, 1994.

WERTSCH, J. La mente en acción. Buenos Aires: Aique, 1999.

Recibido en 18 de enero de 2016.

Aprobado en 07 de marzo de 2017. 